\title{
C-W CELL DECOMPOSITIONS OF SYMMETRIC HOMOGENEOUS SPACES
}

\author{
BY ROBERT HERMANN ${ }^{1}$ \\ Communicated by Hans Samelson, January 4, 1960
}

1. Generalities. Let $G$ be a connected, semi-simple Lie group, $K$ a closed, connected, symmetric subgroup, i.e. there is an automorphism $\sigma: G \rightarrow G$ such that

(a) $\sigma^{2}=$ identity,

(b) $K$ is the connected component of the set of fixed points of $\sigma$.

Let $M$ be the space $G / K$ of right cosets of $K . G$ acts on $G / K$ on the left. Let $G$ and $K$ be the Lie algebras of $G$ and $K$. (We follow Chevalley [4] for the notations of Lie group theory.) Let $\{$,$\} be a$ nondegenerate, symmetric, bilinear form on $G$ that is invariant under Ad $G$ and $\sigma$. Let $M$ be the subspace of $Y \in G$ with $\sigma(Y)=-Y$. Then,

$$
G=K \oplus M, \quad[K, M] \subset M, \quad[M, M] \subset K .
$$

Let $P: G \rightarrow M$ be the projection.

For $X \in G$, let $f_{X}$ be the real-valued function on $M$ such that:

(1.1) for $x=g^{-1} K \in M, g \in G, f_{X}(x)=\{P(\operatorname{Ad} g(X)), P(\operatorname{Ad} g(X))\}$.

Let $C(X)$ be the group of all $g \in G$ with $\operatorname{Ad} g(X)=X$. Let $C(X)$ be its Lie algebra, i.e. the set of all $Y \in G$ with $[X, Y]=0$.

A transvection is an element $g \in G$ such that $\sigma(g)=g^{-1}$. Every $x \in M$ can be written as $g^{-1} K$, with $g$ a transvection (possibly in many ways). We will only use such forms of $x$.

THEOREM 1. $x=g^{-1} K$, with $g$ a transvection, is a critical point of $f_{X}$ if and only if: $\left[\sigma(X), \operatorname{Ad}^{2}(X)\right]=0$. In this case, the orbit of $C(X)$ at $x$, which we denote by $\mathrm{x}$, is a critical manifold for $f_{X}$, nondegenerate in the sense of Bott $[2 ; 3]$. The Hessian of $f_{X}$ at $x$ has the same eigenvalues as the quadratic form on $M$ :

$$
-\left\{\operatorname{Ad} g \operatorname{Ad}(\sigma(X)) \operatorname{Ad}\left(\operatorname{Ad} g^{2}(X)\right) \operatorname{Ad} g(Y), Y\right\} \quad \text { for } Y \in M \text {. }
$$

Another way to consider these results is to look at $M / C(X)$, the orbit space of $M$ under the action of $C(X) . f_{X}$ passes to the quotient to define a function on this space; this theorem says that the critical points of this function are in some sense isolated and nondegenerate.

1 This work was supported in part by Lincoln Laboratory, a center of research operated by Massachusetts Institute of Technology with the joint support of the U.S. Army, Navy and Air Force, and in part by OOR, U. S. Army under contract (number DA-19-020-ORD-3778) with Harvard University. 
THEOREM 2. With the notations of Theorem 1 , the critical orbit $\mathbf{x}$ is again a symmetric homogeneous space if: $\operatorname{Ad} g(C(X)) \subset C(X)$.

Once in possession of these facts, the by-now standard Morse theory of a function on a finite-dimensional manifold will enable one to derive the topological conclusions implied in the title. Of course, computing topological invariants with these decompositions will involve one in the most elaborate parts of Lie algebra theory.

We turn to the most important case, $G$ compact.

2. $G$ compact. We can suppose that the form $\{$,$\} is positive-$ definite. We assume that $\sigma(X)= \pm X$. Let $H$ be a maximal abelian (Cartan) subalgebra of $G$ which contains $X$ and is contained in $C(X)$. Suppose $x=g^{-1} K$, with $g$ a transvection, is a critical point, i.e. Ad $g^{2}(X) \in C(X)$. One shows that there are elements $g^{\prime} \in G, c \in C(X)$, such that:

(a) $g^{2}=g^{\prime} c$,

(b) $\operatorname{Ad} g^{\prime}(H) \subset H$.

Let $W(\boldsymbol{G}, \boldsymbol{H})$ be the group of inner automorphisms of $G$ that map $\boldsymbol{H}$ into itself (the Weyl group). Let $W(\boldsymbol{G}, \boldsymbol{H}, X)$ be the subgroup $W(\boldsymbol{G}, \boldsymbol{H}) \cap \operatorname{Ad} C(X)$. Let $W(\boldsymbol{G}, \boldsymbol{H}) / W(\boldsymbol{G}, \boldsymbol{H}, X)$ be the space of right cosets.

Consider the correspondence $\theta: \mathrm{x} \rightarrow$ (right coset containing Ad $g^{\prime}$ ). Let $F(X)$ denote the set of critical orbits of $f_{X}$. One sees that:

(a) $\theta$ defines a bona-fide map $F(X) \rightarrow W(\boldsymbol{G}, \boldsymbol{H}) / W(\boldsymbol{G}, \boldsymbol{H}, X)$, i.e. this construction is independent of the $x$ and $g$ chosen on the critical orbit.

(b) If, for $x, x_{1} \in F(X), \theta(x)=\theta\left(x_{1}\right)$, then $f_{X}(x)=f_{X}\left(x_{1}\right)$.

(c) Let $K^{\prime}$ be the subgroup of $g \in G$ with $\sigma(g)=g, p: M \rightarrow G / K^{\prime}$ the natural projection. Let $\mathrm{x}, \mathrm{x}_{1} \in F(X)$; if $p(\mathrm{x})=p\left(\mathrm{x}_{1}\right)$, then $\theta(\mathrm{x})=\theta\left(\mathrm{x}_{1}\right)$.

(d) If $f_{X}(x)=0$, then $\theta(x)=$ identity coset of $W(G, H, X)$.

There is interest then in discovering which elements of the Weyl group arise from transvections. We restrict ourselves here to what one can say of a general nature, leaving the detailed application of Lie algebra theory to another work.

Suppose first that $\boldsymbol{H} \cap \boldsymbol{M}$ is a maximal abelian subgroup of $\boldsymbol{M}$. The Weyl group of $M,[1 ; 2]$ is the group of Ad $k$, for $k \in K$, that map $\boldsymbol{H}$ into itself. If $g$ is a transvection such that $\operatorname{Ad} g(H) \subset C$, so is $k g k^{-1}$, for any $k$ whose $\operatorname{Ad} k$ is in the Weyl group of $M$.

Suppose next that $H \subset K$. (This requires that $G / K$ be of positive Euler-Poincaré characteristic.) An Ad $k$, for $k \in K$, such that $\operatorname{Ad} k(H)$ $C H$ defines an element of the Weyl group of $K$. Again, if $g$ is a transvection such that $\operatorname{Ad} g(H) \subset H$, so is $k g k^{-1}$. One should notice in this 
case that if $g$ is a transvection such that $\operatorname{Ad} g \in W(G, H)$, then Ad $g^{2}=$ identity on $H$.

\section{BIBLIOGRAPHY}

1. A. Borel, Lectures on symmetric spaces, Massachusetts Institute of Technology notes, 1958.

2. R. Bott, The stable homotopy of the classical groups, Ann. of Math. vol. 70 (1959) pp. 313-337.

3. R. Bott and H. Samelson, Applications of the theory of Morse to symmetric spaces, Amer. J. Math. vol. 80 (1958) pp. 964-1029.

4. C. Chevalley, Lie groups, I, Princeton, 1946.

HARVARD UNIVERSITY AND

Massachusetts Institute of Technology 